Bundesgesundheitsbl -
Originalien und Übersichtsarbeiten

W. Thierfelder ${ }^{1} \cdot$ Ch.Seher $^{1} \cdot$ R. Dortschy ${ }^{1} \cdot$ S. Engel ${ }^{2}$

${ }^{1}$ Robert Koch-Institut, Berlin • ${ }^{2}$ Dermatologische Universitätsklinik der Charité, Berlin

\section{Abnahme der Spermaqualitåt bei gesunden Männern aus ungewollt kinderlosen Partnerschaften}

\section{Zusammenfassung}

In einer retrospektiven Studie wurden Untersuchungs- und Spermiogrammbefunde von 3821 Männern erhoben. Die Daten stammen aus dem Einzugsbereich der Andrologischen Abteilung der Universitätsklinik Leipzig. Es sollte untersucht werden, ob bei Patienten ohne anamnestische oder klinische Befunde eine Verschlechterung der Spermaqualität innerhalb der Untersuchungsjahre 1985 bis 1996 eingetreten ist. Weiterhin sollten Aussagen über eine mögliche Abhängigkeit der Spermiogrammbefunde vom Geburtsjahr gewonnen werden. Innerhalb der Gruppe der 25-34jährigen ( $n=1650$ ) haben sich von 1985 bis 1996 die absolute Spermienzahl von 195 Mio auf 138 Mio, die Spermienkonzentration von 79,7 auf $52,6 \mathrm{Mio} / \mathrm{ml}$ und der Anteil morphologisch normaler Spermien von $64,6 \%$ auf $59,6 \%$ signifikant $(p<0,001)$ verringert. Mit steigendem Geburtsjahr nahmen sowohl Spermienzahl als auch Spermienkonzentration ab. Die Ursachen für die beobachtete Abnahme bleiben ungeklärt. bwohl die gestörte Fertilität des Mannes kein neues Problem ist, erscheint die Zahl von ca. 1 Million ungewollt kinderlosern Partnerschaften [1] (hochgerechnet auf Deutschland nach der Wiedervereinigung) in der Bundesrepublik doch bedenklich. Nach bisherigen Erfahrungen wird der Anteil der Männer an der ungewollten Kinderlosigkeit mit 40 bis $50 \%$ angesetzt [2]. Es ist seit langem bekannt, daß bestimmte Krankheiten, wie beispielsweise Maldescensus testis, Orchitis, Epididymitis und Varikozele die Fertilität negativ beeinflussen können. Ebensowenig neu ist, daß viele Patienten in die andrologische Sprechstunde kommen, denen keine plausible Ursache zu einer diagnostizierten Fertilitätsstörung zugeordnet werden kann.

In Analogie zu den an Fischen, Reptilien und Vögeln beobachteten auffälligen Störungen der Fortpflanzungsfähigkeit und des Sexualverhaltens, die der Umweltbelastung durch Stoffe mit hormonähnlicher Wirkung zugeschrieben werden, könnten entsprechende Effekte auch für den Menschen gegeben sein. Die wohl bekannteste Zusammenstellung von Chemikalien mit hormonähnlichen Wirkungen wurde 1993 von Colborn zusammengetragen [3]. Für die darin aufgeführten fast 50 Industriechemikalien wurden hormonähnliche Wirkungen nachgewiesen, wobei die Daten unterschiedlichsten experimentellen Be- dingungen entstammten (Übersicht bei [4]). Als Folgerung daraus wurde postuliert, daß diese Chemikalien in Abhängigkeit von der gegebenen Umweltbelastung, also letztlich von der vorliegenden Konzentration und der stoff- oder substratabhängigen Wirkung zumindest in das Hormonsystem von Wildtieren eingreifen könnten [3].

\section{Stand der Forschung}

Der aktuelle Wissensstand läßt es jedoch nicht $\mathrm{zu}$, einen kausalen Zusammenhang zwischen einer Belastung mit Xenohormonen und einer Verschlechterung von Basisparametern der männlichen Fertilität beim Menschen herzustellen bzw. zu beweisen [5]. Hierfür wären die jeweiligen Expositionsdaten aus dem pränatalen bzw. frühen postnatalen Lebensabschnitt erforderlich.

Ein allgemeiner Rückgang der Spermienkonzentration in Abhängigkeit vom Geburtsjahrgang wurde erstmals von Carlsen 1992 [6] in einer Metaanalyse beschrieben (Rückgang von 113 Mio/ml im Jahre 1940 auf $66 \mathrm{Mio} / \mathrm{ml} \mathrm{im}$ Jahre 1990), die in der Folge immer wieder Gegenstand kontrovers geführter Diskussionen wurde. Aber auch neuere

\section{Dr.W.Thierfelder}

Robert Koch-Institut, Postfach 6502 80,

D-13302 Berlin 
Bundesgesundheitsbl -

Gesundheitsforsch - Gesundheitsschutz

1999 - 42: 471-478 @ Springer-Verlag 1999

W. Thierfelder · Ch.Seher · R. Dortschy · S. Engel

Decline of sperm quality in healthy men living in childless partnerships

\section{Summary}

In a retrospective study examination- and spermiogram-data were collected from 3821 men. The data came from the vicinity of the andrological department of the Leipzig university-clinic. It should be examined, whether first-examined patients without anamnestic or abnormal clinical findings did show a deterioration of the sperm-quality within the examination-years 1985 to 1996. Furtheron information should be collected concerning a possible dependence of the spermiogram data from the year of birth. Within the group of the 25 to 34 year old men $(n=1650)$ in the time frame from 1985 until 1996 the absolute sperm count of 195 millions changed to 138 millions, the sperm concentration of 79,7 was reduced to $52,6 \mathrm{Mio} / \mathrm{ml}$ and the fraction of morphological normal sperm was diminished from $64,6 \%$ to $59,6 \%$ (all results with $p<0,001$ ). With increasing birth-year sperm count as well as sperm concentration decreased. The causes for the observed decrease remain unresolved.

\section{Originalien und Übersichtsarbeiten}

Arbeiten belegen einen möglichen Abwärtstrend bei den Basisparametern der männlichen Fertilität $[7,8]$, wobei die nach gegenwärtiger Kenntnis - fertilitätsrelevanten Grenzwerte nicht berührt werden. Interessanterweise existieren hierbei offenbar regionale Unterschiede $[9,10]$. Auch amerikanische Wissenschaftler stellten signifikante Differenzen zwischen den Spermienzahlen in drei Großstädten der USA fest [11]. Demgegenüber stehen Longitudinalstudien, in denen keine Veränderung der Spermienzahlen registriert wurde [12, 13]. In der Literatur finden sich vorrangig Gegenüberstellungen der Ergebnisse von Spermauntersuchungen an zwei unterschiedlichen Zeitpunkten innerhalb einer Kohorte [14-16]. Diese stammen weitgehend von Männern aus kinderlosen Partnerschaften, bzw. von Patienten aus andrologischen Ambulanzen, wie auch die der jüngsten Arbeit aus einer Magdeburger Klinik [17]. Bei diesen Männern wurde eine signifikante $\mathrm{Ab}$ nahme der Gesamtspermienzahl und des prozentualen Anteils normomorpher Spermatozoen registriert.

Um die bei andrologischen Patienten gewonnenen Daten annähernd auf die sogenannte Normalbevölkerung übertragen zu können, müssen alle Patienten mit einem für die Fertilität relevanten anamnestischen Befund und/oder klinischen Befund von einer solchen Untersuchung ausgeschlossen werden.

In der vorliegenden Studie wird über die Entwicklung der spermatologischen Basisparameter andrologischer Patienten zweier andrologischer Zentren mit unterschiedlichen Einzugsgebieten (Leipzig und Berlin) innerhalb eines Zeitraumes von zwölf bzw. 13 Jahren berichtet. Die Herstellung kausaler Zusammenhänge zwischen evtl. festzustellenden Veränderungen der Spermiogrammparameter und diskutierten Umweltfaktoren ist weder beabsichtigt noch im gegebenen Rahmen möglich.

\section{Material und Methoden}

Retrospektiv wurden Untersuchungsund Spermiogrammbefunde von 3821 Männern aus dem Einzugsbereich des
Universitätsklinikums Leipzig (Andrologische Abteilung der Universitätshautklinik, Leiter Prof. H.-J. Glander) der Untersuchungsjahre 1975 bis 1996 erhoben. Davon waren 3818 Datensätze auswertbar.

Anamnestische Daten, die für die Zuordnung entscheidend waren, sind: Maldescensus testis, Mumps mit Hodenaffektion, Leistenbruchoperation, Hydrozelenoperation, Varikozelenoperation bzw. Varikozelentherapie, Genitaltraumen, Orchitis, Epididymitis, endokrine Erkrankungen (Diabetes mellitus), und aktuelle Beschwerden, insbesondere fieberhafte Erkrankungen. Klinische Befunde, die für die Selektion der Patienten entscheidend waren, sind: Hodengröße und Hodenkonsistenz, Veränderungen des Nebenhodens (Verdickung, Verhärtung), Hydrozele und Varikozele.

Spermiogrammparameter, die in die Auswertung einbezogen wurden, sind: Volumen (ml), Spermienkonzentration $\left(10^{6} / \mathrm{ml}\right)$, Gesamtspermienzahl $\left(10^{6}\right)$, Gesamtmotiliät (\%) und der Anteil morphologisch normaler Spermatozoen (\%). Die Labormethoden wurden im Untersuchungszeitraum nicht verändert. Die Untersuchung der Ejakulate erfolgte nach WHO-Standard. Die Karenzzeit vor Probengewinnung betrug fünf Tage. Die Untersuchung und Lagerung der Ejakulate bis zur Untersuchung erfolgte bei Raumtemperatur. Sofern mehr als ein Spermiogrammbefund vorlag, wurde generell der erste erhobene Befund verwendet.

Alle Patienten mit anamnestischen und/oder aktuellen klinischen Befunden wurden lediglich hinsichtlich Erhebung und Darstellung der Basisdaten berücksichtigt ( $n=1113$ von 3818 , Tabelle 1 ), von der weiteren Bearbeitung und Darstellung aber ausgenommen.

Von der weiteren Bearbeitung ausgenommen wurden auch alle Patienten, die bereits anderenorts untersucht und möglicherweise wegen eines normabweichenden Befundes dem andrologischen Zentrum zugewiesen worden waren, so daß sich die Zahl der insgesamt verwendeten Datensätze auf 2437 (erstuntersuchte Patienten) reduzierte (Tabelle 2). Schließlich erfolgte eine Eintei- 


\section{\% ำ ำ}

ถู่ तิ

ํำ กิ

竞

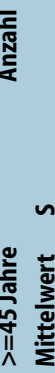

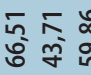

\section{ํㅜㅇㅇㅛ 웅} ชั่ รั ริ

추요

ชิ เึ

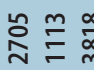

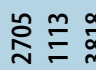

๘ $\infty$

๙

우 눙

侣

$\bar{\sim} \bar{\sim}$

농 $\cong \frac{\infty}{=}$

농 $\frac{m}{=}$

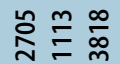

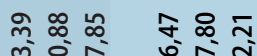

ભิ

ㅇํㅇำ

ำ ㅇํํ 욤

$f \leqslant \frac{6}{\sigma}$

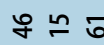

용

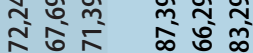

กำ 요

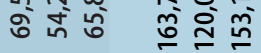

N

ชู

m

\section{}

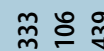

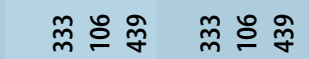

ํำ

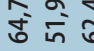

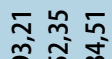

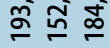

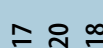

$\approx \infty \approx$

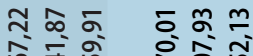

สิ

응 กำ

ำ

ชิ ณ ธ

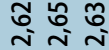

ప̊ః

ป̊요

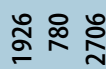

‡̊용

¿ू $\tilde{x}$

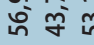

ํํㅇ 옹

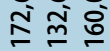

$\simeq \stackrel{2}{ }$

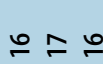

莕望望

盗等

ๆ

守定

m

ชู่ ริธ

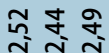

京ำ

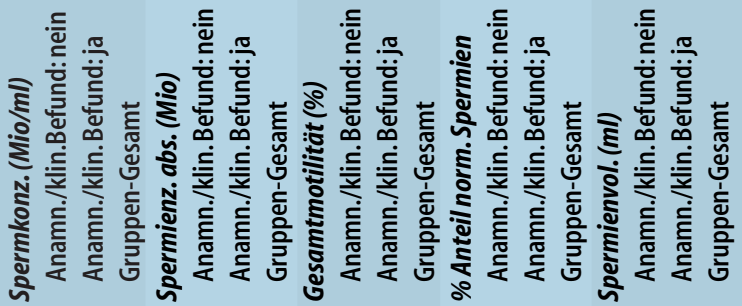

lung der Patienten in vier Altersgruppen: <25 Jahre, 25-34 Jahre, 35-44 Jahre und $\geq 345$ Jahre. Die statistische Analyse konzentrierte sich auf die Altersgruppe der 25-34jährigen ( $n=1926$, Tabelle 1$)$. Wegen zu geringer Patientenzahl in den Untersuchungsjahren 1975 bis 1984 gingen in die statistische Untersuchung nur die Jahre 1985 bis 1996 ein ( $n=1650$, Tabelle 3). Die statistische Untersuchung erfolgte mittels eines SPSS-Programmpakets.

\section{Ergebnisse}

Von den insgesamt 3818 erfaßten Patienten (Tabelle 1) waren 2705, also knapp $71 \%$ ohne anamnestischen und/oder aktuellen klinischen Befund. Zwischen den Patienten mit und denen ohne anamnestischen und/oder aktuellen klinischen Befund wurden bei der Gesamtspermienzahl und der Spermienkonzentration signifikante Unterschiede in allen Altersgruppen registriert (Tabelle 1).

Bei den Patienten ohne anamnestischen und/oder klinischen Befund fällt insbesondere bei der Spermienkonzentration eine deutliche Abnahme mit fallendem Lebensalters auf. So liegt die durchschnittliche Spermienkonzentration in der Gruppe der <25jährigen um $23 \%$ niedriger als in der Gruppe der >45jährigen Männer. Bei der absoluten Spermienzahl beträgt die Differenz sogar $27 \%$. Dieses Phänomen findet sich bei den Patienten mit anamnestischem und/oder klinischem Befund nicht. Allerdings liegt die Fallzahl $(n=46)$ bei den Männern ohne relevanten Befund in der höchsten Altersgruppe deutlich unter der in den beiden anderen Gruppen.

Die Verteilung der Patienten ohne anamnestischen und/oder klinischen Befund in der Altersgruppe 25 bis 34 Jahre $(n=1650)$ auf die Untersuchungsjahre zeigt Tabelle 3.

Die Abb. 1-4 geben die Spermiogrammparameter Gesamtspermienzahl, Spermienkonzentration, Gesamtmotilität der Spermatozoen (WHO-Kategorien a, b und c) und Anteil normomorpher Spermatozoen in dieser Altersgruppe in Abhängigkeit vom Untersuchungsjahr als Scatterplot mit der Regressionsgraden und einem 5\%igen Konfidenzinter- 
Originalien und Übersichtsarbeiten

Tabellen 2a, b

Vergleich von Spermiogrammparametern aller Männer aus den Untersuchungsjahren 1975 bis 1996 ( $n=2437$ ) ohne fertilitätsrelevanten anamnestischen und/oder klinischen Befund vor und nach einer gesetzten Geburtsjahresschwelle

Tabelle 2a

\begin{tabular}{|c|c|c|c|c|c|c|c|c|c|c|c|c|c|c|c|}
\hline \multirow[b]{2}{*}{ Trennschwelle } & \multicolumn{5}{|c|}{ Untersuchungsalter } & \multicolumn{5}{|c|}{ Spermienzahl, abs. (Mio) } & \multicolumn{5}{|c|}{ Spermienkonzentration (Mio/ml) } \\
\hline & Anzahl ${ }^{1)}$ & vor & nach & Diff. & $\mathbf{p}$ & Anzahl & vor & nach & Diff & $\mathbf{p}$ & Anzahl & vor & nach & Diff. & $\mathbf{p}$ \\
\hline 1955 & $376 / 2061$ & 36,9 & 27,7 & 9,2 & $* * *$ & $376 / 2060$ & 187,5 & 164,8 & 22,7 & * & $374 / 2055$ & 77,6 & 66,0 & 11,6 & $* *$ \\
\hline 1956 & $452 / 1985$ & 36,1 & 27,5 & 8,6 & $* * *$ & $452 / 1984$ & 186,8 & 164,1 & 22,7 & * & $450 / 1979$ & 77,2 & 65,6 & 11,6 & $* * *$ \\
\hline 1957 & $549 / 1888$ & 35,1 & 27,4 & 7,7 & $* * *$ & $549 / 1887$ & 184,0 & 163,7 & 20,3 & * & $547 / 1882$ & 75,5 & 65,5 & 10,0 & $* *$ \\
\hline 1958 & $654 / 1783$ & 34,4 & 27,2 & 7,2 & $* * *$ & $654 / 1782$ & 182,7 & 163,0 & 19,7 & * & $652 / 1777$ & 75,4 & 65,0 & 10,4 & $* * *$ \\
\hline 1959 & $798 / 1639$ & 33,5 & 27,0 & 6,5 & $* * *$ & $798 / 1638$ & 185,1 & 160,1 & 25,0 & $* *$ & $796 / 1633$ & 76,6 & 63,5 & 13,1 & $* * *$ \\
\hline 1960 & $968 / 1469$ & 32,7 & 26,8 & 5,9 & $* * *$ & $968 / 1468$ & 186,2 & 156,4 & 29,8 & $* * *$ & $966 / 1463$ & 76,8 & 61,8 & 15,0 & $* * *$ \\
\hline 1961 & $1139 / 1298$ & 32,1 & 26,5 & 5,6 & $* * *$ & $1139 / 1297$ & 182,0 & 156,2 & 25,8 & $* * *$ & $1137 / 1292$ & 74,6 & 61,8 & 12,8 & $* * *$ \\
\hline 1962 & $1321 / 1116$ & 31,5 & 26,3 & 5,2 & $* * *$ & $1321 / 1115$ & 178,2 & 156,5 & 21,7 & $* *$ & $1319 / 1110$ & 73,4 & 61,1 & 12,3 & $* * *$ \\
\hline 1963 & $1511 / 926$ & 31,0 & 26,1 & 4,9 & $* * *$ & $1510 / 926$ & 175,8 & 156,0 & 19,8 & * & $1507 / 922$ & 72,5 & 60,1 & 12,4 & $* * *$ \\
\hline
\end{tabular}

Tabelle 2b

\begin{tabular}{|c|c|c|c|c|c|c|c|c|c|}
\hline \multirow{2}{*}{ Trennschwelle } & \multicolumn{4}{|c|}{ Anteil normomorpher Spermien (\%) } & \multicolumn{5}{|c|}{ Gesamtmotilität (\%) } \\
\hline & Anzahl & vor & nach & Diff. $p$ & Anzahl & vor & nach & Diff. & $\mathbf{p}$ \\
\hline 1955 & $360 / 1962$ & 62,6 & 62,3 & 0,3 & $361 / 1969$ & 73,9 & 74,3 & $-0,4$ & \\
\hline 1956 & $429 / 1893$ & 62,4 & 62,4 & 0,0 & $430 / 1900$ & 73,5 & 74,4 & $-0,9$ & \\
\hline 1957 & $522 / 1800$ & 61,8 & 62,5 & $-0,7$ & $523 / 1807$ & 72,9 & 74,6 & $-1,7$ & * \\
\hline 1958 & $622 / 1700$ & 62,5 & 62,3 & 0,2 & $624 / 1706$ & 73,5 & 74,5 & $-1,0$ & \\
\hline 1959 & $762 / 1560$ & 62,2 & 62,4 & $-0,2$ & $764 / 1566$ & 72,9 & 74,9 & $-2,0$ & * \\
\hline 1960 & $926 / 1396$ & 62,6 & 62,2 & 0,4 & $928 / 1402$ & 73,3 & 74,9 & $-1,6$ & $*$ \\
\hline 1961 & $1090 / 1232$ & 62,8 & 62,0 & 0,8 & $1092 / 1238$ & 73,5 & 74,9 & $-1,4$ & $*$ \\
\hline 1962 & $1258 / 1064$ & 62,7 & 62,0 & 0,7 & $1262 / 1068$ & 73,4 & 75,2 & $-1,8$ & $*$ \\
\hline 1963 & $1433 / 889$ & 62,9 & 61,6 & 1,3 & $1437 / 893$ & 73,6 & 75,3 & $-1,7$ & $*$ \\
\hline
\end{tabular}

$p<0.05=* ;<0.01=* * ;<0.001=* * *$

1) Die Werte vor dem Schrägstrich bedeuten Anzahl der Patienten vor der jeweiligen Trennschwelle, die Werte nach dem Schrägstrich die Anzahl nach der Trennschwelle.

vall wieder. Während bei den Parametern Gesamtspermienzahl, Spermienkonzentration und Anteil morphologisch normaler Spermatozoen die Regressionsgerade eine fallende Tendenz anzeigt, ist bei der Gesamtmotilität ein leichter Anstieg zu verzeichnen.

Auf der Basis einer linearen Regression wurden die Veränderungen für die absolute Spermienzahl, Spermienkonzentration, Gesamtmotilität und den Anteil normomorpher Spermien zwischen den Zeitpunkten 1985 und 1996 berechnet. Die absolute Spermienzahl hat sich im Mittel um 29,2\%, von 195 Mio auf 138 Mio verringert. Die Spermienkonzentration fiel in dieser Zeit im Mittel um $34 \%$, von $79,7 \mathrm{Mio} / \mathrm{ml}$ auf 52,6 $\mathrm{Mio} / \mathrm{ml}$. Das ergibt rechnerisch einen
Abfall von 2,4 Mio/ml im Jahr. Die Gesamtmotilität stieg im Mittel von 72,1\% auf $77,3 \%$, während der Anteil morphologisch normaler Spermien im Mittel von $64,6 \%$ auf $59,6 \%$ zurückging. Alle Unterschiede sind signifikant $(p<0,01)$.

Um den Einfluß von Untersuchungsalter und Untersuchungsjahr auf obige Spermienparameter festzustellen, wurde ein multiples lineares Regressionsmodell angewandt. Auf die absolute Spermienzahl hat das Untersuchungsjahr einen signifikanten negativen Einfluß von 5,2 Mio pro Untersuchungsjahr unter Berücksichtigung des Untersuchungsalters. Hingegen hat das Alter zum Zeitpunkt der Untersuchung in diesem Modell keinen signifikanten Einfluß. Ein ähnliches Ergebnis zeigt sich für die Spermienkonzentration: Der signifikante negative Zusammenhang mit dem Untersuchungsjahr beträgt hierbei 2,5 Mio/ml pro Untersuchungsjahr. Auch dieser Parameter steht mit dem Untersuchungsalter in keiner signifikant abhängigen Beziehung. Auf die Gesamtmotilität hat das Untersuchungsjahr einen signifikant positiven Einfluß von 0,45\%, auf den Anteil morphologisch normaler Spermien dagegen einen signifikant negativen Einfluß von $0,45 \%$ pro Untersuchungsjahr. Das Untersuchungsalter spielt auch für diese Meßgrößen keine Rolle. Die vermutete Altersabhängigkeit der Spermiogrammparameter wurde auch unabhängig vom Untersuchungsjahr (Tabelle 1), näher untersucht. Dazu wurden alle gesunden, 


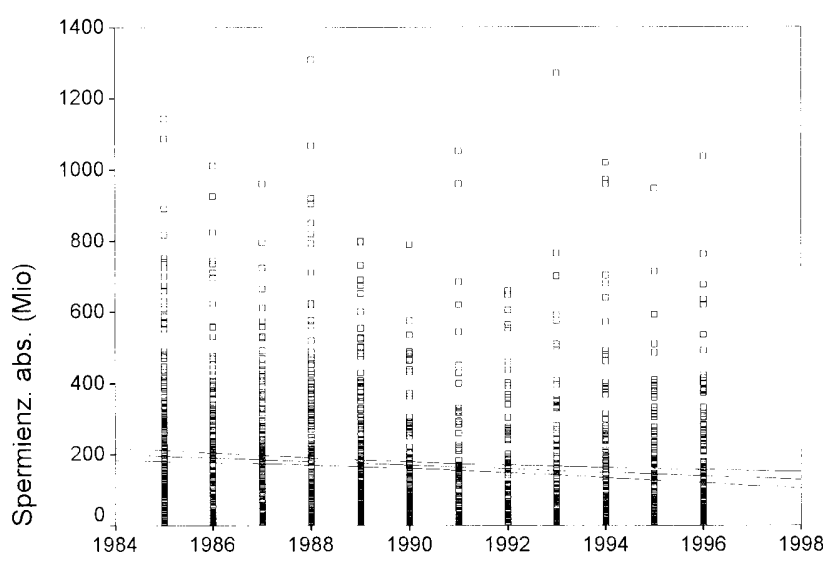

$1 \quad$ Untersuchungsjahr

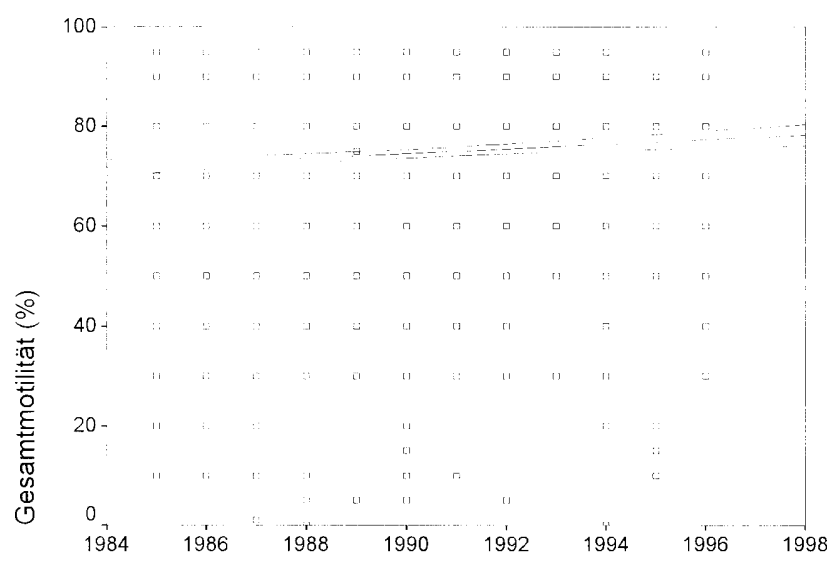

3 Untersuchungsjahr

Abb. $1 \Delta$ Gesamtspermienzahl (Mio) der Männer ohne relevanten anamnestischen und/oder aktuellen klinischen Befund in der Altersgruppe 25 bis 34 Jahre ( $n=1650$ ) in Abhängigkeit vom Untersuchungsjahr Abb. $\triangle \Delta$ Gesamtmotilität der Spermien (\%) der Männer ohne relevanten anamnestischen und/oder aktuellen klinischen Befund in der Altersgruppe 25 bis 34 Jahre $(n=1650)$ in Abhängigkeit vom Untersuchungsjahr

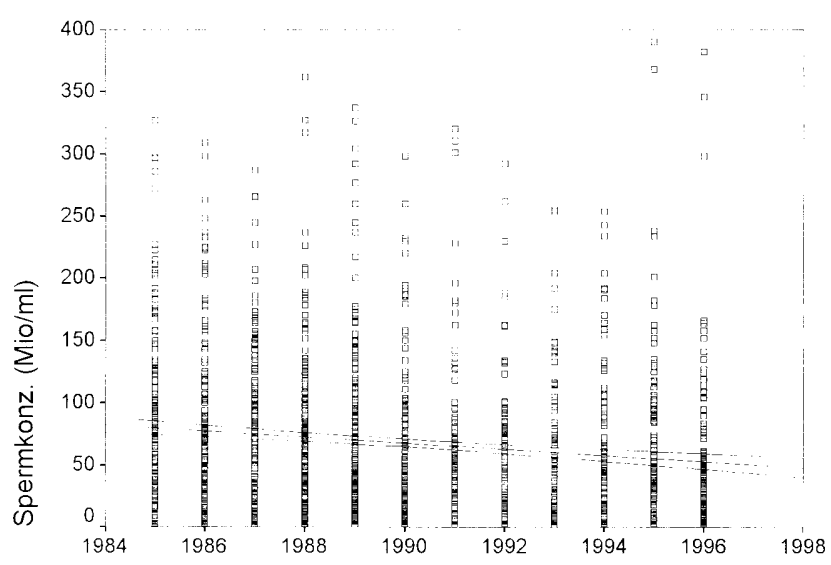

$2 \quad$ Untersuchungsjahr

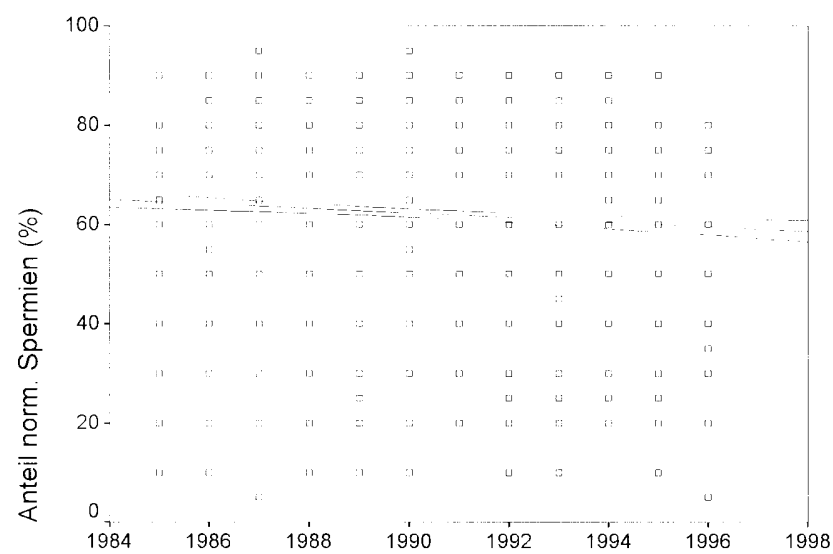

$4 \quad$ Untersuchungsjahr

Abb. $2 \triangle$ Spermienkonzentration (Mio/ml) der Männer ohne relevanten anamnestischen und/oder aktuellen klinischen Befund in der Altersgruppe 25 bis 34 Jahre $(n=1650)$ in Abhängigkeit vom Untersuchungsjahr Abb.4 $\Delta$ Anteil normomorpher Spermien (\%) bei Männern ohne relevanten anamnestischen und/oder aktuellen klinischen Befund in der Altersgruppe 25 bis 34 Jahre ( $n=1650$ ) in Abhängigkeit vom Untersuchungsjahr anamnestisch unauffälligen erstuntersuchten Probanden aus dem gesamten Untersuchungszeitraum (1975-1996) entweder einer Gruppe vor oder einer Gruppe nach einem bestimmten Geburtsjahr zugeteilt und miteinander verglichen. Die Trennschwellen wurden schrittweise vom Jahresbeginn 1955 bis zum Jahresbeginn 1963 verschoben (Tabelle $2 \mathrm{a}$ und 2b). 2437 Probanden (alle Männer ohne fertilitätsrelevanten anamnestischen und/oder klinischen Befund) gingen in diesen Vergleich ein.

Ein fallweiser Ausschluß wegen einzelner fehlender Meßwerte erfolgte nicht, daher ergeben sich bei den untersuchten Variablen unterschiedliche $\mathrm{Pa}$ - tientenzahlen. Bei den Parametern Gesamtspermienzahl und Spermienkonzentration sind die Differenzen zwischen der jeweils jüngeren und der jeweils älteren Gruppe unabhängig von der Festsetzung der Trennschwelle signifikant. Der größte Unterschied findet sich bei Setzen der Trennschwelle auf den Jahresbeginn 1960. So verringert sich die Spermienkonzentration bei den nach 1960 geborenen im Vergleich $\mathrm{zu}$ den vor diesem Zeitpunkt geborenen Patienten um 20\% und die absolute Spermienzahl um 16\%. Der Anteil normomorpher Spermien ändert sich über den gesamten Beobachtungszeitraum nicht wesentlich. Bei der Gesamtmotili- tät haben die jüngeren Jahrgänge bessere Werte.

Die tendenzielle Verschlechterung von Gesamtspermienzahl und Spermienkonzentration in Abhängigkeit vom Geburtsjahr wird auch in den Abbildungen 5 und 6 deutlich. Bei der Gesamtmotilität und dem Anteil normomorpher Spermien läßt sich dieser Trend nicht ausmachen (Abb.7 und 8). Die Selektion der Patienten für diese Untersuchung erfolgte analog der TrennschwellenDarstellung (Tabelle 2a und 2b). Die einzelnen Geburtsjahrgänge wurden in Zweiergruppen zusammengefaßt.

Eine Abhängigkeit der Befunde vom Lebensalter der Patienten zum 


\begin{tabular}{|c|c|c|}
\hline Untersuchungs-Jahr & Anzahl & Prozent \\
\hline 1985 & 175 & 10,6 \\
\hline 1986 & 168 & 10,2 \\
\hline 1987 & 152 & 9,2 \\
\hline 1988 & 185 & 11,2 \\
\hline 1989 & 190 & 11,5 \\
\hline 1990 & 141 & 8,5 \\
\hline 1991 & 92 & 5,6 \\
\hline 1992 & 92 & 5,6 \\
\hline 1993 & 89 & 6,4 \\
\hline 1994 & 109 & 6,6 \\
\hline 1995 & 123 & 7,5 \\
\hline 1996 & 134 & 8,1 \\
\hline Gesamt & 1650 & 100 \\
\hline
\end{tabular}

Zeitpunkt der Untersuchung läßt sich nicht nachweisen, zumal das mittlere Kinderwunschalter der Männer dieser Klientel von 1985 bis 1996 nur um etwa 36 (alle Altersgruppen, gesund, erstuntersucht) bzw. 14 Monate (Altersgruppe 25-34 Jahre, gesund, erstuntersucht) zugenommen hat (Abb.10 und 11).

\section{Diskussion}

Bei den Parameter Gesamtspermienzahl und Spermienkonzentration ergibt sich bei den 25- bis 34jährigen eine fallende Tendenz (Abb. 1 und 2). Diese Beobachtung findet sich in der Literatur mehrfach $[7,8,14-16]$. Allerdings werden von den Autoren überwiegend nur zwei Zeitpunkte miteinander verglichen, wobei sich größere Differenzen ergeben als in der eigenen Untersuchung. Die Magdeburger Arbeitsgruppe um Glöckner [17] stellte einen Abfall der Spermienkonzentration von im Mittel $48 \mathrm{Mio} / \mathrm{ml}$ auf $26 \mathrm{Mio} / \mathrm{ml}$ innerhalb von 20 Jahren (1974-1994) fest. Für diese Verringerung der Konzentration auf $53 \%$ des Ausgangswertes wird von den Autoren eine jährliche Abnahme von 2,1\% errechnet. Diese Ergebnisse korrellieren im wesentlichen mit den von uns ermittelten Werten. Obwohl unsere Klientel im Gegensatz zum Magdeburger Kollektiv einer Selektion (ausschließlich Patienten ohne fertilitätsrelevanten anamnestischen und/oder aktuellen klinischen Befund) unterzogen wurde, fiel die mittlere Spermienkonzentration in den beobachteten elf Jahren um ca. $34 \%$. Eine Abhängigkeit der Befunde vom Lebensalter der Patienten zum Zeitpunkt der Untersuchung läßt sich nicht nachweisen, zumal das mittlere Kinderwunschalter der Männer dieser Klientel von 1985 bis 1996 nur um etwa 36 (alle Altersgruppen, gesund, erstuntersucht) bzw. 14 Monate (Altersgruppe 25-34 Jahre, gesund, erstuntersucht) zugenommen hat (Abb. 10 und 11).

Die insgesamt höheren Mittelwerte im Vergleich zur Magdeburger Studie sind am ehesten auf die Selektion an- nehmbar Gesunder, d.h. Männer ohne fertilitätsrelevante Erkrankungen in unserer Studie zurückzuführen. Allerdings kämen ursächlich auch methodische Unterschiede zwischen den Laboren in Betracht [18]. Normalerweise verschlechtern sich mit zunehmendem Lebensalter die Spermiogrammbefunde. Spermienzahl und Spermienkonzentration nehmen jedoch nicht nur mit dem späteren Untersuchungsjahr sondern auch mit dem späterem Geburtsjahr ab (Abb. 5 und 6), wobei in dieser Betrachtung das Alter zum Zeitpunkt der Untersuchung unberücksichtigt blieb. Das starke Absinken der Mittelwerte bei hoher Varianz ab Geburtsjahr 1972 in unserer Untersuchungsklientel ist dabei offenbar auf die geringe Fallzahl zurückzuführen. Ähnliche Resultate werden sowohl von Irvine [17] als auch von Glöckner [19] beschrieben. Da das Untersuchungsalter mit späterem Geburtsjahr abnimmt (Abb. 9), es sich dagegen mit dem Untersuchungsjahr erhöht, müßte die Verschlechterung von Spermienzahl und Spermienkonzentration vom Untersuchungsalter der Patienten weitgehend unabhängig sein. Damit bleiben die Ursachen für die beobachtete Abnahme der Spermienzahlen weiterhin ungeklärt.

Danksagung Wir danken Herrn Professor Glander, Andrologische Abteilung der Universitäts-Hautklinik Leipzig für die Bereitstellung der Daten.
Abb. 5 Mittelwerte und 95\% Konfidenzintervall der Gesamtspermienzahl (Mio) in Abhängigkeit vom Geburtsjahr (jeweils zwei Jahre zusammengefaßt)

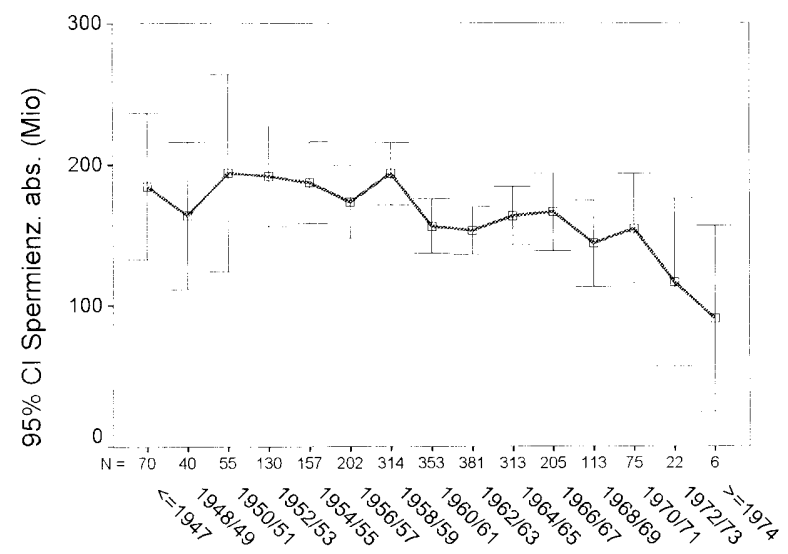

Geburtsjahresgruppen 


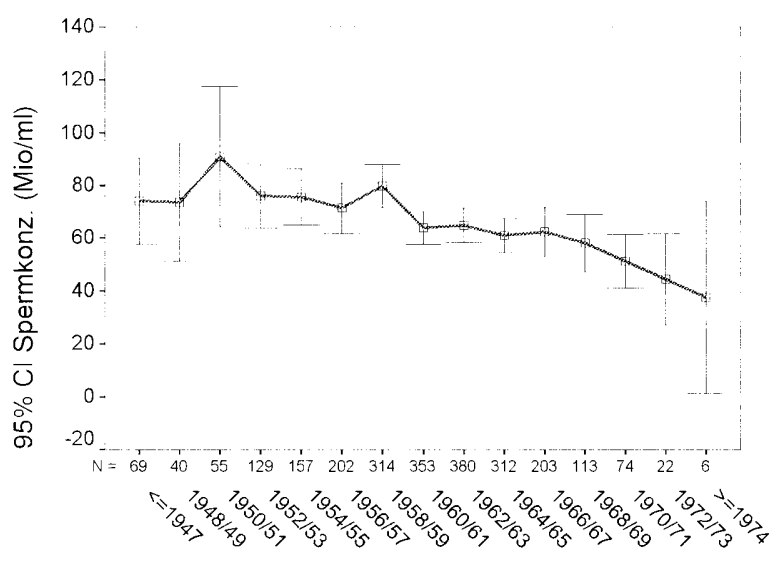

6

Geburtsjahresgruppen

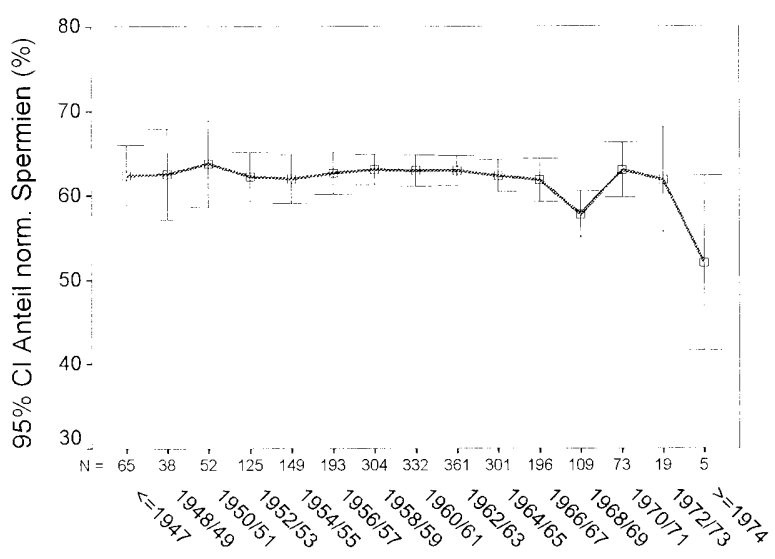

8

Geburtsjahresgruppen

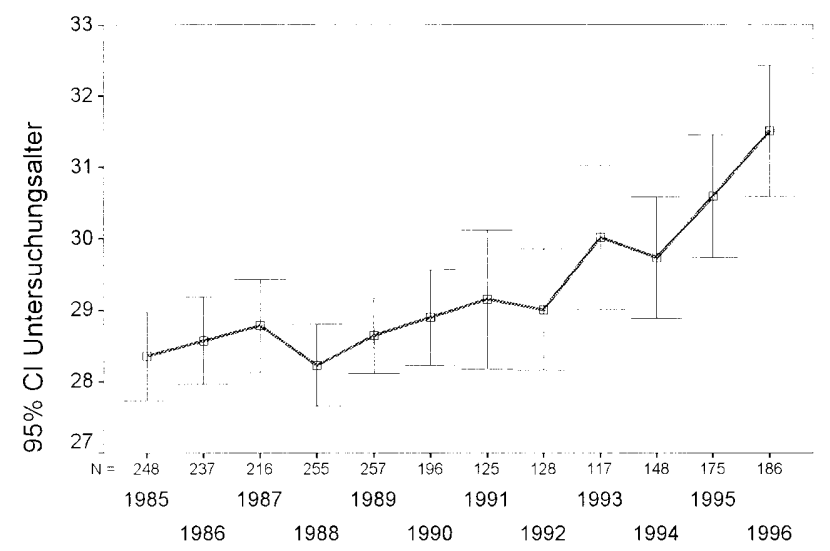

10

Untersuchungsjahr

Abb. $6 \Delta$ Mittelwerte und 95\% Konfidenzintervall der Spermienkonzentration (Mio/ml) in Abhängigkeit vom Geburtsjahr (jeweils zwei Jahre zusammengefaßt)

Abb. $8 \Delta$ Mittelwert und 95\% Konfidenzintervall für des Anteils normomorpher Spermien (\%) in Abhängigkeit vom Geburtsjahr (jeweils zwei Jahre zusammengefaßt)

Abb. $10 \Delta$ Mittelwert und 95\% Konfidenzintervall des Lebensalters von allen (erstuntersuchten) Männern ohne fertilitätsrelevanten anamnestischen und/oder klinischen Befund ( $n=2437)$ in den Untersuchungsjahren 1985 bis 1996

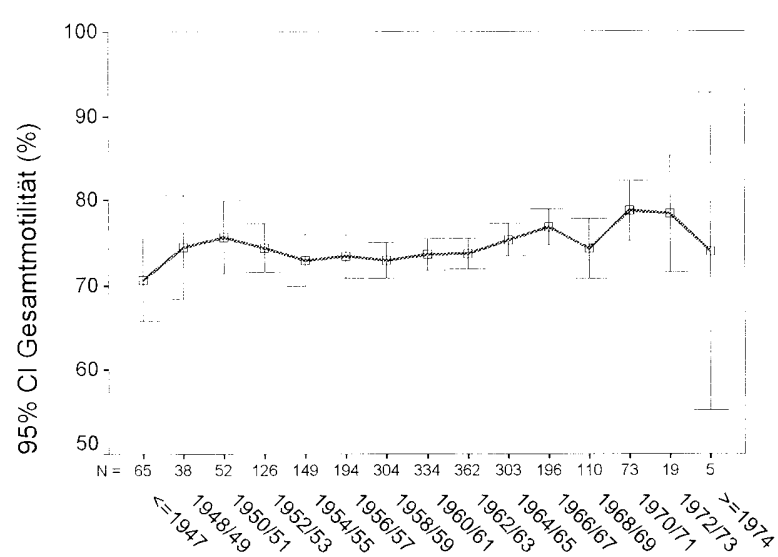

7 Geburtsjahresgruppen

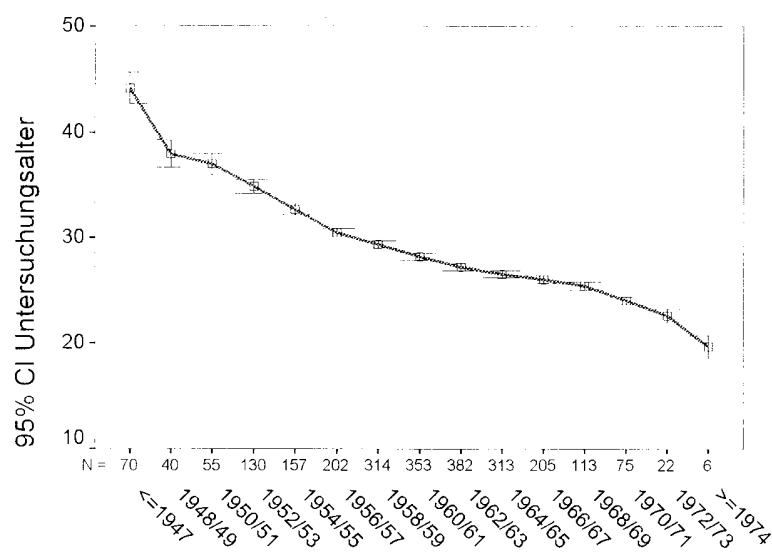

9 Geburtsjahresgruppen

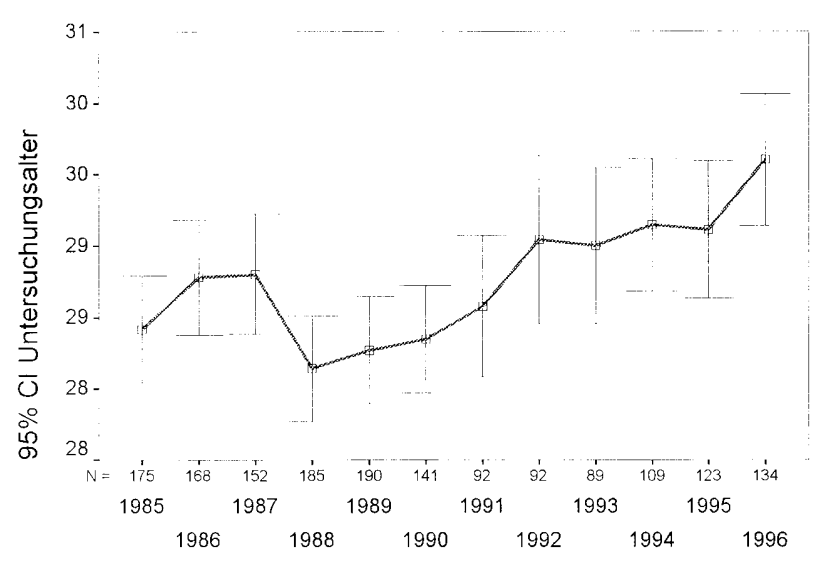

11 Untersuchungsjahr

Abb.7 $\triangle$ Mittelwert und 95\% Konfidenzintervall der Gesamtmotilität (\%) in Abhängigkeit vom Geburtsjahr (jeweils zwei Jahre zusammengefaßt)

Abb.9 $\triangle$ Mittelwert und 95\% Konfidenzintervall des Untersuchungsalters (Jahre) in den Geburtsjahresgruppen (jeweils zwei Jahre zusammengefaßt)

Abb. $11 \Delta$ Mittelwert und 95\% Konfidenzintervall des Lebensalters der 25- bis 34jährigen (erstuntersuchten) Männer ohne fertilitätsrelevanten anamnestischen und/oder klinischen Befund $(n=1650)$ in den Untersuchungsjahren 1984 bis 1996

Bundesgesundheitsbl - Gesundheitsforsch - Gesundheitsschutz 6.99 


\section{Literatur}

1. Bruckert $E$ (1991) How frequent is unintentional childlessness in Germany? Andrologia 23: $245-250$

2. WHO Task Force on the Diagnosis and Treatment of Infertility (1987) Towards more objectivity in diagnosis and management of male infertility. Int J Androl [Suppl 7]

3. Colborn T, vom Saal FS, Soto AM (1993) Developmental effects of endocrine-disrupting chemicals in wildlife and humans. Environ Health Perspect 101:378-384

4. Thierfelder W, Mehnert WH, Laußmann D, Arndt D, Reineke HH (1995) Der Einfluß umweltrelevanter östrogener oder östrogenartiger Substanzen auf das Reproduktionssystem. Bundesgesundhbl 38:338-341

5. Greim H (1998) Hormonähnlich wirkende Stoffe in der Umwelt - Einführung und Sachstand. Bundesgesundhbl 41:326-329

6. Carlsen E, Giwercman A, Keiding N, Skakkebaek NE (1992) Evidence for decreasing quality of semen during past 50 years. BMJ 305:609-613

7. van Waeleghem K, et al (1996) Deterioration of sperm quality in young healthy belgian men. Human Reproduction 11:101-105

8. Irvine $S$ (1994) Falling sperm quality. BMJ 309:476

9. Auger J, Kunstmann JM, Czyglik F, Jouannet P (1995) Decline in semen quality among fertile men in Paris during past 20 years. $N$ Engl J Med 332:281-285

10. Bujan $\mathrm{L}$, et al (1996) Time series analysis of sperm concentration in fertile men in Toulouse, France between 1977 and 1992. BMJ 312:471-472

11. Fish $\mathrm{H}$, et al (1996) Semen analysis in $\mathbf{1 2 8 3}$ men from the United States over a 25-year period: no decline in quality. Fertility and Sterility 65 : 1009-1014

12. Wittmaack $F$, et al (1992) Longitudinal study of semen quality in Wisconsin men over one decade. Wisconsin Med J 91:477-479

13. Fish $\mathrm{H}$, et al (1995) Semen analysis in 632 men over a 25-year period: no change in quality. J Urology 153 [Suppl]:323A

14. Bendvold $E$ (1989) Semen quality in Norwegian men over a 20 year period. Int J Fert 34: 401-404

15. Bendvold E, et al (1991) Depressed semen quality in swedish men from barren couples: a study over three decades. Archives of Andrology 26: 189-194

16. Ayala C, Steinberger E, Smith DP (1996) The influence of semen analysis parameters on the fertility potential of infertile couples. Journal of Andrology 17:718-725

17. Glöckner D, Gacvert K, Kleinstein J (1998) Declining sperm quality in men of childless couples. Andrologia 30:55

18. Lerchl A, Nieschlag E (1996) Gibt es eine Spermienkrise? Deutsches Ärzteblatt 93: A2465-2468

19. Irvine S, Cawood E, Richardson D, MacDonald E, Aitken J (1996) Evidence of deteriorating semen quality in the United Kingdom: birth cohort study in $\mathbf{5 7 7}$ men in Scotland over 11 years. BMJ 312:467-471

\section{G. Winter}

Die Prüfung der Freisetzung von gentechnisch veränderten Organismen. Recht und Genehmigungspraxis.

Reihe: Umweltbundesamt Berichte, Band 4/98. Berlin: Erich Schmidt Verlag, 1998. 159 S., (ISBN 3-503-04397-7) kart. DM 48,-

Bei dem Buch handelt es sich um ein Gutachten, das von Winter (Forschungsstelle für Europäisches Umweltrecht, Universität Bremen) unter Mitwirkung von fünf weiteren Autoren (s.u.) im Auftrag des Umweltbundesamtes erstellt wurde. Hinter dem sehr allgemein gehaltenen Titel des Buches verbirgt sich hauptsächlich eine Analyse des Aspektes schädlicher Einwirkungen durch gentechnisch veränderte Organismen, insbesondere wie er im Rahmen von Genehmigungsverfahren in Deutschland, England und Dänemark als materieller Maßstab der Genehmigung verwendet wird. Im ersten Kapitel behandelt Jörgensen auf der Grundlage von Rechtstexten, Rechtsprechung und Literatur die materiellen Voraussetzungen der Freisetzungsgenehmigung. Im zweiten Kapitel wird von Fisahn an Hand einer Fallstudie die Genehmigungspraxis in Deutschland untersucht. Dem wird im dritten und vierten Kapitel der Vollzug für die Genehmigung von Freisetzungsvorhaben in England (Macrory und Purdy) bzw. in Dänemark (Anker) gegenübergestellt. Daran schließt sich im fünften Kapitel (Winter) eine wertende Zusammenfassung an, aus der Reformüberlegungen entwickelt werden.

Obwohl die Abfolge der Kapitel eine Gesamtschau der Argumente vermittelt, könnte dem Leser dieses Buches der gelegentliche Blick in das deutsche Gentechnikgesetz von Nutzen sein, um nicht blindlings den mitunter eigenwil- ligen Interpretationen der Autoren folgen zu müssen. Als Beispiele seien angeführt:

a) Der vom Autor eingeführten Kategorie von Freisetzungen zu gewerblichen Zwecken fehlt die textliche Entsprechung im deutschen Gentechnikrecht. Spezielle Anforderungen an Freisetzungsvorhaben zu gewerblichen Zwecken (bzw.zu experimentellen Zwecken) bestehen von Gesetzes wegen grundsätzlich nicht.

b) Schwer nachvollziehbar ist auch die Vorstellung, daß gewerbliche(mitgedacht ist: massenhafte) Freisetzungsvorhaben auch nach dem Inverkehrbringen erfolgen können. Hiergegen ist an die Systematik des Step-by-StepVorgehens (im Idealfall vom Labor- und Gewächshausexperiment über kleinräumige Freisetzungsexperimente zu großräumigen Freisetzungsexperimenten und schließlich zum Inverkehrbringen) zu erinnern.

c) Die Analyse des Genehmigungsbescheides zu einem Fallbeispiel (aus dem Jahr 1995!) verkennt, daß die Bewertung der Antragsunterlagen zu dem Ergebnis geführt hat, daß nach dem Stand der Wissenschaft keine schädlichen Einwirkungen auf die in $\S 1$ GenTG bezeichneten Rechtsgüter zu erwarten sind. Insofern handelt es sich bei den in den Nebenbestimmungen auferlegten Maßnahmen um Vorsorgemaßnahmen, die u.a. geeignet sind, eine dem Maßstab des vorgesehenen Versuchs entsprechende, hinreichende Begrenzung der gentechnisch veränderten Organismen zu gewährleisten.

Insgesamt kann das Buch dennoch empfohlen werden, wenn es das Anliegen des Lesers ist, einen schnellen Überblick über die Schwierigkeiten zu bekommen, die mit der Definition des Schadensbegriffes (insbesondere im Hinblick auf Ökosysteme) verbunden sind. 